\title{
Boneh-Franklin Identity Based Encryption Revisited
}

\author{
David Galindo \\ Institute for Computing and Information Sciences \\ Radboud University Nijmegen \\ P.O.Box 90106500 GL, Nijmegen, The Netherlands. d.galindo@cs.ru.nl
}

\begin{abstract}
The first practical identity based encryption (IBE) scheme was proposed by Boneh and Franklin in [BF03]. In this work we point out that there is a flawed step in the security reduction exhibited by the authors. Fortunately, it is possible to fix it without changing the scheme or the underlying assumption.

In the second place, we introduce a variant of the seminal IBE scheme which allows a more efficient security reduction. The new scheme is simpler, and has more compact ciphertexts than Boneh-Franklin's proposal, while keeping the computational cost.

Finally, we observe that the flawed step pointed out here is present in several works, and that our techniques can be applied to obtain tighter reductions for previous relevant schemes.
\end{abstract}

Keywords: provable security, identity-based encryption, exact security, bilinear maps.

\section{Introduction}

The concept of Identity Based Encryption (IBE) was proposed by Shamir in [Sha85], aimed at simplifying certificate management in e-mail related systems. The idea is that an arbitrary string such as an e-mail address or a telephone number could serve as a public key for an encryption scheme. Once a user $U$ receives a communication encrypted using its identity $I D_{U}$, the user authenticates itself to a Private Key Generation Center (KGC) from which it obtains the corresponding private key $d_{\mathrm{ID}_{U}}$.

The problem was not satisfactorily solved until the work by Boneh and Franklin [BF03]. They proposed formal security notions for IBE systems and designed a fully functional secure IBE scheme using bilinear maps. The security is based on a variant of the Computational Diffie-Hellman assumption, called Bilinear Diffie-Hellman assumption. This scheme and the tools developed in its design have been successfully applied in numerous cryptographic settings, transcending by far the identity based cryptography framework.

On the other hand, an important concern when exhibiting a security reductionist proof is that of the efficiency of the reduction. One of the goals pursued 
is to preserve as much as possible the strength of the underlying hard problem which is used in the protocol's design. An inefficient security reduction would imply the use of larger key sizes to attain a given security level.

Our contributions. In the first place, we show there is a flawed step in the security reduction exhibited in [BF03] for the scheme proposed for chosen ciphertext security. Fortunately, the reduction can be changed without modifying the original scheme or the underlying hard problem used to state the security. The efficiency of the new security reduction is a bit worse than the previous one. This is just another example in which a well-known and widely used construction turns out to have an unnoticed flawed security reduction.

In the second place, we modify the scheme by Boneh and Franklin towards obtaining a more efficient security reduction. Indeed, it is possible to show a tighter security reduction for a modified scheme which uses one less random oracle. The new proposal also presents more compact ciphertexts than the original scheme.

Finally, since Boneh-Franklin IBE scheme has been used as a building block for numerous protocols, the corrections and improvements we present here are likely to be applied to further schemes. For instance, this is the case for the schemes in [GS02,HL02,Gen03,AP03,YFDL04,CC05].

\section{Preliminaries}

We begin by fixing some notation. If $A$ is a non-empty set, then $x \leftarrow A$ denotes that $x$ has been uniformly chosen in $A$. If $A$ is a finite set, then $|A|$ denotes its cardinality.

\subsection{Definitions for IBE schemes}

Identity based encryption (IBE). An IBE is specified by four probabilistic polynomial time (PPT) algorithms:

Setup takes a security parameter $1^{\ell}$ and returns the system parameters params and master-key. The system parameters include the description of sets $\mathcal{M}, \mathcal{C}$, which denote the set of messages and ciphertexts respectively. params is publicly available, while the master-key is kept secret by the KGC.

Extract takes as inputs params, master-key and an arbitrary string ID $\in\{0,1\}^{*}$ and returns a private key $d_{\mathrm{ID}}$ to the user with identity ID. This must be done over a secure channel, since $d_{\mathrm{ID}}$ enables to decrypt ciphertexts under the identity ID.

Encrypt takes as inputs params, ID $\in\{0,1\}^{*}$ and $M \in \mathcal{M}$. It returns a ciphertext $C \in \mathcal{C}$.

Decrypt takes as inputs params, $C \in \mathcal{C}$ and a private key $d_{\mathrm{ID}}$, and it returns $M \in \mathcal{M}$ or rejects. 
Chosen ciphertext security. An IBE scheme is said to have indistinguishability against an adaptive chosen ciphertext attack (IND-ID-CCA) if any PPT algorithm $\mathcal{A}$ has a negligible advantage in the following game:

Setup The challenger takes a security parameter $1^{\ell}$ and runs the Setup algorithm. It gives params to the adversary. It keeps the master-key to itself.

Phase 1 The adversary issues queries of the form

- Extraction query $\left\langle\mathrm{ID}_{i}\right\rangle$. The challenger runs algorithm Extract to generate the private key $d_{i}$ corresponding to $\mathrm{ID}_{i}$. It sends $d_{i}$ to the adversary.

- Decryption query $\left\langle\mathrm{ID}_{i}, C_{i}\right\rangle$. The challenger generates the private key $d_{i}$. It then runs Decrypt to decrypt $C_{i}$ under $\mathrm{ID}_{i}$.

These queries may be asked adaptively, that is, each query may depend on the answers obtained to the previous queries.

Challenge The adversary outputs equal length plaintexts $M_{0}, M_{1} \in \mathcal{M}$ and an identity $I D_{c h}$. The only constraint is that the private key for $I D_{c h}$ was not requested in Phase 1 . The challenger picks $\beta \leftarrow\{0,1\}$ and sets $C=$ Encrypt (params, $\left.\mathrm{ID}_{\mathrm{ch}}, M_{\beta}\right)$. It sends $C$ to the adversary.

Phase 2 The adversary issues extraction and decryption queries as in Phase 1, with the restriction $\left\langle\mathrm{ID}_{i}\right\rangle \neq\left\langle\mathrm{ID}_{\mathrm{ch}}\right\rangle$ and $\left\langle\mathrm{ID}_{i}, C_{i}\right\rangle \neq\left\langle\mathrm{ID}_{\mathrm{ch}}, C\right\rangle$.

Guess The adversary outputs a guess $\beta^{\prime} \in\{0,1\}$.

Such an adversary is called an IND-ID-CCA adversary $\mathcal{A}$, and its advantage \left. is defined ${\operatorname{as~} \operatorname{Adv}_{\mathcal{E}, \mathcal{A}}^{\mathrm{C}} \mathrm{CA}}_{1} 1^{\ell}\right)=\left|\operatorname{Pr}\left[\beta=\beta^{\prime}\right]-1 / 2\right|$.

Similarly, indistinguishability against passive adversaries (IND-ID-CPA) can also be defined. In this case, the game between the challenger and the adversary is similar to the IND-ID-CCA case, but disallowing decryption queries. The advantage of an adversary in this game is defined as $\operatorname{Adv}_{\mathcal{E}, \mathcal{A}}^{\mathrm{CPA}}\left(1^{\ell}\right)=\left|\operatorname{Pr}\left[\beta=\beta^{\prime}\right]-1 / 2\right|$.

Definition 1. An IBE system $\mathcal{E}$ is secure under chosen ciphertext attacks (resp. chosen plaintext attacks) if for any probabilistic polynomial time IND-ID-CCA (resp. IND-ID-CPA) adversary $\mathcal{A}$ the function $\operatorname{Adv}_{\mathcal{E}, \mathcal{A}}^{\mathrm{CCA}}\left(1^{\ell}\right)\left(\right.$ resp. $\left.\operatorname{Adv}_{\mathcal{E}, \mathcal{A}}^{\mathrm{CPA}}\left(1^{\ell}\right)\right)$ is negligible.

\subsection{Bilinear maps and bilinear groups}

Let $\mathbb{G}_{1}, \mathbb{G}_{2}$ and $\mathbb{G}_{T}$ be finite abelian groups in which the discrete logarithm is believed to be hard. We use additive notation for $\mathbb{G}_{1}, \mathbb{G}_{2}$ whereas multiplicative notation is used for $\mathbb{G}_{T}$. Thus, $\mathbb{G}_{1}^{*}=\mathbb{G}_{1} \backslash\left\{O_{1}\right\}$ and $\mathbb{G}_{T}^{*}=\mathbb{G}_{T} \backslash\left\{1_{T}\right\}$, where $O_{1}$ and $1_{T}$ are the identity elements in $\mathbb{G}_{1}$ and $\mathbb{G}_{T}$ respectively. By a pairing or bilinear map we will refer to a non-degenerate bilinear function $\widehat{e}: \mathbb{G}_{1} \times \mathbb{G}_{2} \rightarrow \mathbb{G}_{T}$. In some protocols the existence of a computable isomorphism $\psi: \mathbb{G}_{2} \rightarrow \mathbb{G}_{1}$ is assumed. In particular, this implies that $\psi\left(a P_{2}\right)=a \psi\left(P_{2}\right)$. By a bilinear group we refer to a tuple $\left(\mathbb{G}_{1}, \mathbb{G}_{2}, \mathbb{G}_{T}, \widehat{e}, \psi\right)$ with the properties described above.

Bilinear maps are usually implemented using the Weil or modified Tate pairings on an elliptic curve. In general, the elements in $\mathbb{G}_{1}$ allow more compact representation than those in $\mathbb{G}_{2}$. In the following it is assumed that $\left|\mathbb{G}_{1}\right|=$ 
$\left|\mathbb{G}_{2}\right|=\left|\mathbb{G}_{T}\right|=p$, where $p$ is prime; $\mathbb{G}_{1}, \mathbb{G}_{2}$ are cyclic groups generated by $P_{1}, P_{2}$ respectively and $\psi\left(P_{2}\right)=P_{1}$. In this context, the map $\widehat{e}$ is non-degenerate if and only if $\widehat{e}\left(P_{1}, P_{2}\right) \neq 1_{G_{T}}$. We refer to [BF03] for further details.

Bilinear Diffie-Hellman (BDH) Problem on $\left(\mathbb{G}_{1}, \mathbb{G}_{2}\right)$. Given $a P_{2}, b P_{2} \in \mathbb{G}_{2}^{*}$ and $c P_{1} \in \mathbb{G}_{1}^{*}$, where $P_{2} \leftarrow \mathbb{G}_{2}^{*}, P_{1}=\psi\left(P_{2}\right), a, b, c \leftarrow \mathbb{Z}_{p}^{*}$; compute $W=$ $\widehat{e}\left(P_{1}, P_{2}\right)^{a b c} \in \mathbb{G}_{T}$.

We say that an algorithm $\mathcal{B}(t, \varepsilon)$ breaks $\mathrm{BDH}$ on $\left(\mathbb{G}_{1}, \mathbb{G}_{2}\right)$ if it runs in time at most $t$ and has advantage at least $\varepsilon$, that is,

$$
\left.\operatorname{Pr}\left[\mathcal{B}\left(P_{2}, a P_{2}, b P_{2}, c P_{1}\right)=\widehat{e}\left(P_{1}, P_{2}\right)^{a b c}\right)\right] \geq \varepsilon,
$$

where the probability is taken over the random choices of the parameters, and the random bits of $\mathcal{B}$.

Bilinear Decision Diffie-Hellman (BDDH) Problem on $\left(\mathbb{G}_{1}, \mathbb{G}_{2}\right)$. Let $a P_{2}, b P_{2} \in \mathbb{G}_{2}^{*}, c P_{1} \in \mathbb{G}_{1}^{*}$, and $T \leftarrow \mathbb{G}_{T}$, where $P_{2} \leftarrow \mathbb{G}_{2}^{*}, P_{1}=\psi\left(P_{2}\right), a, b, c \leftarrow$ $\mathbb{Z}_{p}^{*}$. We say that an algorithm $\mathcal{B}(t, \varepsilon)$ breaks BDDH on $\left(\mathbb{G}_{1}, \mathbb{G}_{2}\right)$ if it runs in time at most $t$ and

$\left|\operatorname{Pr}\left[\mathcal{B}\left(P_{2}, a P_{2}, b P_{2}, c P_{1}, \widehat{e}\left(P_{1}, P_{2}\right)^{a b c}\right)=1\right]-\operatorname{Pr}\left[\mathcal{B}\left(P_{2}, a P_{2}, b P_{2}, c P_{1}, T\right)=1\right]\right| \geq \varepsilon$,

where the probability is computed over the random choices of the parameters, and the random bits of $\mathcal{B}$. Hereafter, the distribution on the left side is called $B D H$ distribution and is denoted by $\mathcal{P}_{B D H}$, while the distribution on the right is called random $B D H$ distribution and is denoted by $\mathcal{R}_{B D H}$.

\section{Security proof of Boneh-Franklin identity based encryption scheme revisited}

In this section we consider the identity based encryption (IBE) scheme by Boneh and Franklin [BF03]. In the first place, we point out and fix a flaw in the security reduction given by the authors. In repairing the proof, we do not need to change the security assumption neither the specification of the scheme. However, the security reduction is a bit worse than the original one.

\subsection{Boneh-Franklin IBE scheme}

We will not directly use the original description of the BF scheme, because it is phrased with bilinear group pairs where $\mathbb{G}_{1}=\mathbb{G}_{2}$, so we must adapt their scheme to the more general case $\mathbb{G}_{1} \neq \mathbb{G}_{2}$. In choosing how to use $\mathbb{G}_{1}$ and $\mathbb{G}_{2}$, we preferred to minimize the length of the ciphertexts. This means we use $\mathbb{G}_{2}$ as the set of private keys and then ciphertexts are elements in $\mathbb{G}_{1}^{*} \times\{0,1\}^{n}$. Here follows the description of the BF scheme, which is called Full-Ident in [BF03]. 


Full-Ident
Setup. Let $\left(\mathbb{G}_{1}, \mathbb{G}_{2}, G_{T}, \widehat{e}, \psi\right)$ a bilinear group. Choose a gen-
erator $P_{2} \leftarrow \mathbb{G}_{2}$ and set $P_{1}=\psi\left(P_{2}\right)$. Next pick $s \leftarrow \mathbb{Z}_{p}^{*}$
and set $Q_{p u b}=s P_{2} \in \mathbb{G}_{2}^{*}, P_{p u b}=s P_{1} \in \mathbb{G}_{1}^{*}$. Choose crypto-
graphic hash functions $H_{1}:\{0,1\}^{*} \rightarrow \mathbb{G}_{2}^{*}, H_{2}: \mathbb{G}_{T} \rightarrow\{0,1\}^{n}$,
$H_{3}:\{0,1\}^{n} \times\{0,1\}^{n} \rightarrow \mathbb{Z}_{p}^{*}, H_{4}:\{0,1\}^{n} \rightarrow\{0,1\}^{n}$. The message space
is $\mathcal{M}=\{0,1\}^{n}$ and the ciphertext space is $\mathcal{C}=\mathbb{G}_{1}^{*} \times\{0,1\}^{n} \times\{0,1\}^{n}$.
Extract. For a given string ID $\in\{0,1\}^{*}$, compute $Q_{\mathrm{ID}}=H_{1}(\mathrm{ID}) \in \mathbb{G}_{2}^{*}$
and set the private key $d_{\mathrm{ID}}$ to be $d_{\mathrm{ID}}=s Q_{\mathrm{ID}} \in \mathbb{G}_{2}^{*}$.
Encrypt. To encrypt $M \in\{0,1\}^{n}$ under identity ID, compute $Q_{\mathrm{ID}}=$
$H_{1}(\mathrm{ID}) \in \mathbb{G}_{2}^{*}$, choose $\sigma \leftarrow\{0,1\}^{n}$, set $r=H_{3}(\sigma, M) \in \mathbb{Z}_{p}^{*}$ and finally
$C=\left\langle r P_{1}, \sigma \oplus H_{2}\left(g_{\mathrm{ID}}^{r}\right), M \oplus H_{4}(\sigma)\right\rangle$ where $g_{\mathrm{ID}}=\widehat{e}\left(P_{p u b}, Q_{\mathrm{ID}}\right) \in \mathbb{G}_{T}$.
Decrypt. Let $C=\langle U, V, W\rangle \in \mathcal{C}$ be a ciphertext under the identity
ID. To decrypt $C$ using the private key $d_{\mathrm{ID}} \in \mathbb{G}_{2}^{*}$ do:
1. Compute $V \oplus H_{2}\left(\widehat{e}\left(U, d_{\mathrm{ID}}\right)\right)=\sigma$.
2. Compute $W \oplus H_{4}(\sigma)=M$.
3. Set $r=H_{3}(\sigma, M)$. Check that $U=r P$. If not, reject the ciphertext.
4. Output $M$.

This completes the description of Full-Ident. This IBE scheme is sound since

$$
\widehat{e}\left(U, d_{\mathrm{ID}}\right)=\widehat{e}\left(r P_{1}, s Q_{\mathrm{ID}}\right)=\widehat{e}\left(P_{1}, Q_{\mathrm{ID}}\right)^{s r}=\widehat{e}\left(P_{p u b}, Q_{\mathrm{ID}}\right)^{r}=g_{\mathrm{ID}}^{r}
$$

In [BF03] it is proven that the above scheme is IND-ID-CCA secure under the BDH assumption in the Random Oracle model. That scheme uses Fujisaki and Okamoto transformation [FO99] from a one-way encryption scheme into an IND-CCA encryption scheme in the ROM (we refer to [BDPR98] for public key encryption security notions). If we denote by $E_{\mathrm{pk}}(M, r)$ the encryption of $M$ using the random bits $r$ under the public key pk, the transformation by Fujisaki and Okamoto is the hybrid scheme ${ }^{1}$

$$
E_{\mathrm{pk}}^{h y}(M)=\left\langle E_{\mathrm{pk}}\left(\sigma, H_{3}(\sigma, M)\right), H_{4}(\sigma) \oplus M\right\rangle
$$

where $\sigma$ is random and $H_{3}, H_{4}$ are random oracles. To decrypt $\left(C_{1}, C_{2}\right)$, one first obtains $\sigma^{\prime}$ decrypting $C_{1}$ using the original scheme, next computes $M^{\prime}$ and finally checks if $E_{\mathrm{pk}}\left(\sigma^{\prime}, H_{3}\left(\sigma^{\prime}, M\right)\right)=C_{1}$. If this is so, outputs $M$; otherwise outputs reject.

Two additional schemes are needed in order to exhibit the security proof in [BF03]. These schemes are not IBE schemes but merely public key encryption schemes. They are called BasicPub and BasicPub ${ }^{h y}$. Here follows the description of

\footnotetext{
${ }^{1}$ In the case where the symmetric encryption scheme is the one-time pad.
} 


\section{BasicPub}

KeyGen. Let $\left(\mathbb{G}_{1}, \mathbb{G}_{2}, G_{T}, \widehat{e}, \psi\right)$ a bilinear group. Choose a generator $P_{2} \leftarrow \mathbb{G}_{2}$ and set $P_{1}=\psi\left(P_{2}\right)$. Next pick $s \leftarrow \mathbb{Z}_{p}^{*}, Q \leftarrow \mathbb{G}_{2}^{*}$ and set $Q_{\text {pub }}=s P_{2} \in \mathbb{G}_{2}^{*}, P_{\text {pub }}=s P_{1} \in \mathbb{G}_{1}^{*}$. Choose $H_{2}: \mathbb{G}_{T} \rightarrow\{0,1\}^{n}$. Then $\mathcal{M}=\{0,1\}^{n}$ and $\mathcal{C}=\mathbb{G}_{1}^{*} \times\{0,1\}^{n}$. The public key is

$$
\mathrm{pk}=\left\langle p, \mathbb{G}_{1}, \mathbb{G}_{2}, \mathbb{G}_{T}, \widehat{e}, P_{1}, P_{2}, P_{p u b}, Q_{p u b}, Q, H_{2}\right\rangle .
$$

The private key is $\mathrm{sk}=d=s Q \in \mathbb{G}_{2}^{*}$.

Encrypt. To encrypt $M \in\{0,1\}^{n}$ choose $r \leftarrow \mathbb{Z}_{p}^{*}$ and set the ciphertext to be

$$
C=\left\langle r P_{1}, M \oplus H_{2}\left(g^{r}\right)\right\rangle \quad \text { where } \quad g=\widehat{e}\left(P_{p u b}, Q\right) \in \mathbb{G}_{T}
$$

Decrypt. Let $C=\langle U, V\rangle \in \mathcal{C}$ be a ciphertext under the public key pk. To decrypt $C$ using the private key $d \in \mathbb{G}_{2}^{*}$, compute $V \oplus H_{2}(\widehat{e}(U, d))=M$.

Finally, the scheme BasicPub ${ }^{h y}$ is the result of applying Fujisaki-Okamoto transformation (1) to the above scheme. The security reduction for Full-Ident scheme under the BDH assumption follows the diagram below

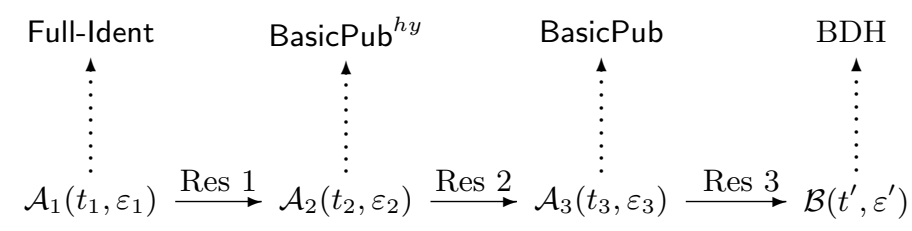

The following results are shown in [BF03]. Hereafter, $q_{E}, q_{D}, q_{H_{i}}$ denote the number of extraction, decryption and random oracle $H_{i}$ queries respectively.

Result 1 Let $\mathcal{A}_{1}$ an IND-ID-CCA adversary that has advantage $\varepsilon_{2}$ against Full-Ident making at most $q_{E}, q_{D}$ and $q_{H_{1}}$ queries. Then there is an IND-CCA adversary $\mathcal{A}_{2}$ that has advantage at least $\frac{\varepsilon_{1}}{e\left(1+q_{E}+q_{D}\right)}$ against BasicPub ${ }^{\text {hy }}$. Its running time is $t_{2} \leq t_{1}+c_{\mathbb{G}_{2}}\left(q_{D}+q_{H_{1}}+q_{E}\right)$, where $c_{\mathbb{G}_{2}}$ denotes the time of computing a random multiple in $\mathbb{G}_{2}$.

Result 2 Let $\mathcal{A}_{2}$ an IND-CCA adversary that has advantage $\varepsilon_{2}$ against BasicPub ${ }^{\text {hy }}$ making at most $q_{D}, q_{H_{3}}$ and $q_{H_{4}}$ queries. Then there is an IND-CPA adversary $\mathcal{A}_{3}$ that has advantage at least $\frac{1}{2\left(q_{H_{3}}+q_{H_{4}}\right)}\left[\left(\varepsilon_{2}+1\right)(1-2 / p)^{q_{D}}-1\right]$ against BasicPub. Its running time is $t_{3} \leq t_{2}+\mathcal{O}\left(\left(q_{H_{3}}+q_{H_{4}}\right) \cdot(n+\log p)\right)$.

Result 3 Let $\mathcal{A}_{3}$ an IND-CPA adversary that has advantage $\varepsilon_{3}$ against BasicPub making at most $q_{\mathrm{H}_{2}}$ queries. Then there is an algorithm $\mathcal{B}$ breaking the $B D H$ problem on $\left(\mathbb{G}_{1}, \mathbb{G}_{2}\right)$ with advantage at least $\frac{2 \varepsilon_{3}}{q_{H_{2}}}$ and running time $t^{\prime} \approx t_{3}$. 
In order to come up with the total concrete security, we can bound any $q_{H_{i}}$ with a single $q_{H}$, and assume that $q_{E}=q_{D}$, since extraction and decryption operations have roughly the same computational complexity. Then, taking the above reductions, we obtain that the $\mathrm{BF}$ scheme is $\left(t_{1}, q_{H}, q_{D}, \varepsilon_{1}\right)$ IND-ID-CCA secure if the BDH problem on $\left(\mathbb{G}_{1}, \mathbb{G}_{2}\right)$ is

$$
\left.\left(t_{1}+c_{\mathbb{G}_{1}}\left(2 q_{D}+q_{H}\right)\right)+2 q_{H}(n+\log p), \frac{\varepsilon_{1}}{8 e q_{H}^{2} q_{D}}\right) \text {-secure. }
$$

Therefore, the security reduction is far from tight, mainly because of the $q_{H}^{2} q_{D}$ factor relating the advantages against the scheme and the underlying problem.

\subsection{A flaw in the security reduction}

In this section we point out a flaw in the reduction used to state Result 1, which is Lemma 4.6 in [BF03].

The goal of that reduction is to construct an IND-CCA adversary $\mathcal{B}$ with advantage $\varepsilon / e\left(1+q_{E}+q_{D}\right)$ against BasicPub ${ }^{h y}$ by using an IND-ID-CCA adversary $\mathcal{A}$ with advantage $\varepsilon$ against Full-Ident. $\mathcal{B}$ receives a public key

$$
K_{p u b}=\left\langle p, \mathbb{G}_{1}, \mathbb{G}_{2}, \mathbb{G}_{T}, \widehat{e}, P_{1}, P_{2}, P_{p u b}, Q, Q_{p u b}, H_{2}, H_{3}, H_{4}\right\rangle
$$

from its challenger. Then $\mathcal{B}$ simulates the challenger for $\mathcal{A}$ as follows:

Setup $\mathcal{B}$ gives $\mathcal{A}$ the parameters $\left\langle p, \mathbb{G}_{1}, \mathbb{G}_{2}, \mathbb{G}_{T}, \widehat{e}, P_{1}, P_{2}, P_{\text {pub }}, Q_{\text {pub }}, H_{1}, H_{2}, H_{3}, H_{4}\right\rangle$, where $H_{1}$ is an oracle controlled by $\mathcal{B}$ as indicated in the following:

$H_{1}$-queries To respond to $\mathcal{A}$ queries, algorithm $\mathcal{B}$ maintains a list $H_{1}^{\text {list }}$ of tuples $\left\langle\mathrm{ID}_{i}, Q_{i}, b_{i}, c_{i}\right\rangle$ as explained below. When $\mathcal{A}$ queries $H_{1}$ at an unrepeated $\mathrm{ID}_{i}, \mathcal{B}$ generates a random coin $c_{i} \in\{0,1\}$ such that $\operatorname{Pr}\left[c_{i}=0\right]=\delta$, and a random $b_{i} \leftarrow \mathbb{Z}_{p}^{*}$. If $c_{i}=0$ it computes $Q_{i}=b_{i} P_{2} \in \mathbb{G}_{2}^{*}$, and if $c_{i}=1$ it computes $Q_{i}=b_{i} Q \in \mathbb{G}_{2}^{*}$. Finally, $\mathcal{B}$ adds the tuple $\left\langle\mathrm{ID}_{i}, Q_{i}, b_{i}, c_{i}\right\rangle$ to the $H_{1}^{\text {list }}$ and sends $H_{1}\left(\mathrm{ID}_{i}\right)=Q_{i}$ to $\mathcal{A}$.

The idea is that tuples with $c_{i}=0$ enable $\mathcal{B}$ to answer private key queries for identity $\mathrm{ID}_{i}$, while $\mathcal{B}$ can only take profit of $\mathcal{A}$ 's advantage when $\mathcal{A}$ chooses a challenge identity $\mathrm{ID}_{\mathrm{ch}}$ such that $c_{\mathrm{ch}}=1$.

Phase 1 - Extraction queries When $\mathcal{A}$ asks for the private key associated to $\mathrm{ID}_{i}, \mathcal{B}$ runs the algorithm for responding $H_{1}$-queries and gets $H_{1}\left(\mathrm{ID}_{i}\right)=$ $Q_{i}$, where $\left\langle\mathrm{ID}_{i}, Q_{i}, b_{i}, c_{i}\right\rangle$ is the corresponding entry in $H_{1}^{\text {list }}$. If $c_{i}=1$ then $\mathcal{B}$ aborts the game and the attack against BasicPub ${ }^{h y}$ failed. Otherwise, $c_{i}=0$ and therefore $Q_{i}=b_{i} P_{2}$. It turns out that $d_{i}$ can be computed as $d_{i}:=b_{i} Q_{p u b}$, since by definition $d_{i}=s Q_{i}$. Finally, $\mathcal{B}$ gives $d_{i}$ to algorithm $\mathcal{A}$.

Phase 1 - Decryption queries $\mathcal{B}$ answers to a decryption query $\left\langle\mathrm{ID}_{i}, C_{i}\right\rangle$ as follows. It runs $H_{1}$-queries algorithm and let $\left\langle\mathrm{ID}_{i}, Q_{i}, b_{i}, c_{i}\right\rangle \in H_{1}^{\text {list }}$. If $c_{i}=$ 0 , then $\mathcal{B}$ retrieves the private key $d_{i}$ and decrypts $C_{i}$ using the decryption algorithm. If $c_{i}=1$, then $Q_{i}=b_{i} Q$. Recall that the unknown private key is $d_{i}=s Q_{i}=s b_{i} Q$. Set $C_{i}^{\prime}=\left\langle b_{i} U_{i}, V_{i}, W_{i}\right\rangle$, where $C_{i}=\left\langle U_{i}, V_{i}, W_{i}\right\rangle$. Then, the 
authors claim that the Full-Ident decryption of $C_{i}$ is equal to the BasicPub ${ }^{h y}$ decryption of $C_{i}^{\prime}$. The reason given is that

$$
\widehat{e}\left(b_{i} U_{i}, d\right)=\widehat{e}\left(b_{i} U_{i}, s Q\right)=\widehat{e}\left(U_{i}, s b_{i} Q\right)=\widehat{e}\left(U_{i}, s Q_{i}\right)=\widehat{e}\left(U_{i}, d_{i}\right),
$$

which implies that the values $\sigma$ and $M$ obtained by decrypting $C_{i}$ with Full-Ident and by decrypting $C_{i}^{\prime}$ with BasicPub ${ }^{h y}$ are equal. However, BasicPub ${ }^{h y}$ will output the reject symbol when decrypting $C_{i}^{\prime}$ with overwhelming probability. To see this, remember that $b_{i} U_{i}=b_{i} r_{i} P_{1}$, and at least $b_{i} \leftarrow \mathbb{Z}_{p}^{*}$, which implies that $b_{i} r$ is uniformly random in $\mathbb{Z}_{p}^{*}$. On the other hand, we have that $H_{3}$ is a random oracle not controlled by $\mathcal{B}$. These facts imply that $H_{3}(\sigma, M) \neq b_{i} r$ with probability $1-1 / p$, and therefore the decryption algorithm of BasicPub ${ }^{h y}$ will reject the ciphertext. Thereby, we can not use the decryption oracle for BasicPub ${ }^{h y}$ to decrypt ciphertexts under any $\mathrm{ID}_{i}$ such that $H_{1}\left(\mathrm{ID}_{i}\right) \neq Q$. Therefore, the reduction in [BF03] is not valid.

\subsection{Fixing the security reduction}

Due to the ciphertext integrity checking in FO transformation [FO99], we can only answer decryption queries $\left\langle\mathrm{ID}_{i}, C_{i}\right\rangle$ such that:

- $H_{1}\left(\mathrm{ID}_{i}\right)=b_{i} P_{2}$, since we can use the private key $d_{i}$, or

- $H_{1}\left(\mathrm{ID}_{i}\right)=Q$, since in this case, the decryption of $C_{i}$ under such $\mathrm{ID}_{i}$ is equal to the decryption of $C_{i}$ by BasicPub ${ }^{h y}$.

This remark enables us to fix the flawed reduction shown above. In the following we describe the new answers delivered by $\mathcal{B}$.

Setup As in Section 3.2.

$H_{1}$-queries Before initializing $H_{1}^{\text {list }}, \mathcal{B}$ selects at random $j \leftarrow\left\{1, \ldots, q_{H_{1}}\right\}$. When $\mathcal{A}$ queries $H_{1}$ at $\mathrm{ID}_{i}$, algorithm $\mathcal{B}$ proceeds as follows: if $i \neq j$, it picks $b_{i} \leftarrow \mathbb{Z}_{p}^{*}$, sets $Q_{i}=b_{i} P_{2}$, adds $\left\langle\mathrm{ID}_{i}, Q_{i}, b_{i}\right\rangle$ to the list and gives back $Q_{i}$ to $\mathcal{A}$. If $i=j$, it sets $Q_{j}=Q$, adds $\left\langle\mathrm{ID}_{i}, Q_{i},{ }^{*}\right\rangle$ to the list and sends $Q_{j}$ to $\mathcal{A}$. Here ${ }^{*}$ denotes a special symbol. Note that the outputs of $H_{1}$ are uniformly distributed in $\mathbb{G}_{2}^{*}$ and independent of $\mathcal{A}^{\prime} s$ current view, since $Q$ is unknown to $\mathcal{A}$ and is uniformly distributed in $\mathbb{G}_{2}^{*}$.

Phase 1 - Extraction queries When $\mathcal{A}$ asks for the private key for $\mathrm{ID}_{i}, \mathcal{B}$ runs the algorithm for responding $H_{1}$-queries and gets $H_{1}\left(\mathrm{ID}_{i}\right)=Q_{i}$, where $\left\langle\mathrm{ID}_{i}, Q_{i}, b_{i}\right\rangle$ is the corresponding entry in $H_{1}^{\text {list }}$. If $i=j$, then $\mathcal{B}$ aborts the game and the attack against BasicPub ${ }^{h y}$ failed. Otherwise, it sets $d_{i}:=b_{i} Q_{p u b}$. Finally, $\mathcal{B}$ gives $d_{i}$ to algorithm $\mathcal{A}$.

Phase 1 - Decryption queries $\mathcal{B}$ answers to a decryption query $\left\langle\mathrm{ID}_{i}, C_{i}\right\rangle$ as follows. It runs $H_{1}$-queries algorithm and let $\left\langle\mathrm{ID}_{i}, Q_{i}, b_{i}\right\rangle \in H_{1}^{\text {list }}$. If $i \neq j$, then $\mathcal{B}$ retrieves the private key $d_{i}$ and decrypts $C_{i}$ using the decryption algorithm. If $i=j$, then $Q_{i}=Q$, and the decryption of $\left\langle\mathrm{ID}_{j}, C_{j}\right\rangle$ is the same as the decryption of $C_{j}$ under BasicPub ${ }^{h y}$. Then, $\mathcal{B}$ asks its challenger to decrypt $C_{j}$ and relays the answer to $\mathcal{A}$. 
Challenge $\mathcal{A}$ outputs a public key $\mathrm{ID}_{\mathrm{ch}}$ and two equal length plaintexts $M_{0}, M_{1}$. Algorithm $\mathcal{B}$ proceeds as follows. If $\mathrm{ID}_{\mathrm{ch}} \neq \mathrm{ID}_{j}$, it aborts the game and the attack against BasicPub $^{h y}$ failed. Otherwise, it sends $M_{0}, M_{1}$ to its own challenger and gets back $C$, the encryption of $M_{\beta}$ for a random bit $\beta$ under BasicPub ${ }^{h y}$. Finally, $\mathcal{B}$ relays $C$ to $\mathcal{A}$, which is an also encryption of $M_{\beta}$ under $I_{\mathrm{ch}}$ for Full-Ident.

Phase 2 - Extraction queries Algorithm $\mathcal{B}$ proceeds as in Phase 1, except for the extraction query for $I D_{c h}$, which is rejected.

Phase 2 - Decryption queries Algorithm $\mathcal{B}$ proceeds as in Phase 1, except for the decryption query $\left\langle\mathrm{ID}_{\mathrm{ch}}, C_{\beta}\right\rangle$, which is rejected.

Guess Algorithm $\mathcal{A}$ outputs a guess $\beta^{\prime}$ for $\beta$. $\mathcal{B}$ outputs $\beta^{\prime}$ as its guess.

Using this algorithm $\mathcal{B}$, we are able to state the following:

Result 4 Let $\mathcal{A}$ an IND-ID-CCA adversary that has advantage $\varepsilon$ against Full-Ident making at most $q_{E}, q_{D}$ and $q_{H_{1}}$ queries. Then there is an IND-CCA adversary $\mathcal{B}$ that has advantage at least $\frac{\varepsilon}{q_{H_{1}}}\left(1-\frac{q_{E}}{q_{H_{1}}}\right) \approx \frac{\varepsilon}{q_{H_{1}}}$ against BasicPub ${ }^{\text {hy }}$. Its running time is $t_{2} \leq t_{1}+c_{\mathbb{G}_{2}}\left(q_{D}+q_{H_{1}}+q_{E}\right)$, where $c_{\mathbb{G}_{2}}$ denotes the time of computing a random multiple in $\mathbb{G}_{2}$.

Proof: See Appendix A.

Therefore, joining Results 2, 3 and 4, an IND-ID-CCA advantage $\epsilon_{1}$ against Full-Ident is turned into an algorithm with advantage roughly $\varepsilon_{1} /\left(q_{H}^{3}\right)$ in solving the $\mathrm{BDH}$ problem. Compared to the original flawed reduction, where the advantage obtained against $\mathrm{BDH}$ was roughly $\varepsilon_{1} /\left(q_{H}^{2} q_{D}\right)$, the new reduction is a bit worse, since in general $q_{D} \ll q_{H}$. In the next section we show a modification of Full-Ident which allows a tighter security reduction.

\section{A new identity based encryption scheme with improved tightness}

In this section we design a new IBE scheme using the scheme Basic-Ident from the previous section and a second general transformation also due to Fujisaki and Okamoto [FO00]. This conversion starts from an IND-CPA encryption scheme and builds an IND-CCA scheme in the ROM. If we denote by $E_{\mathrm{pk}}(M, r)$ the encryption of $M$ using the random bits $r$ under the public key pk, with set of messages $\mathcal{M}=\{0,1\}^{n}$, set of coins $\mathcal{R}$ and set of ciphertexts $\mathcal{C}$, the new transformation is the scheme

$$
E_{\mathrm{pk}}^{\text {hyNew }}(M)=E_{\mathrm{pk}}(M \| r, H(M \| r))
$$

where $M \| r \in\{0,1\}^{n-k_{0}} \times\{0,1\}^{k_{0}}$ and $H:\{0,1\}^{*} \rightarrow \mathcal{R}$ is a hash function. Then, $\mathcal{M}^{\text {hyNew }}=\{0,1\}^{n-k_{0}}, \mathcal{R}^{\text {hyNew }}=\{0,1\}^{k_{0}}$ and $\mathcal{C}^{\text {hyNew }}=\mathcal{C}$. To decrypt $C$, one first obtains $M^{\prime} \| r^{\prime}$ using the original decryption algorithm, and next checks if $E_{\mathrm{pk}}\left(M^{\prime} \| r^{\prime}, H\left(M^{\prime} \| r^{\prime}\right)\right)=C$. If this is so, outputs $M$; otherwise outputs 
reject.

Let us describe the new IBE scheme thereby obtained.

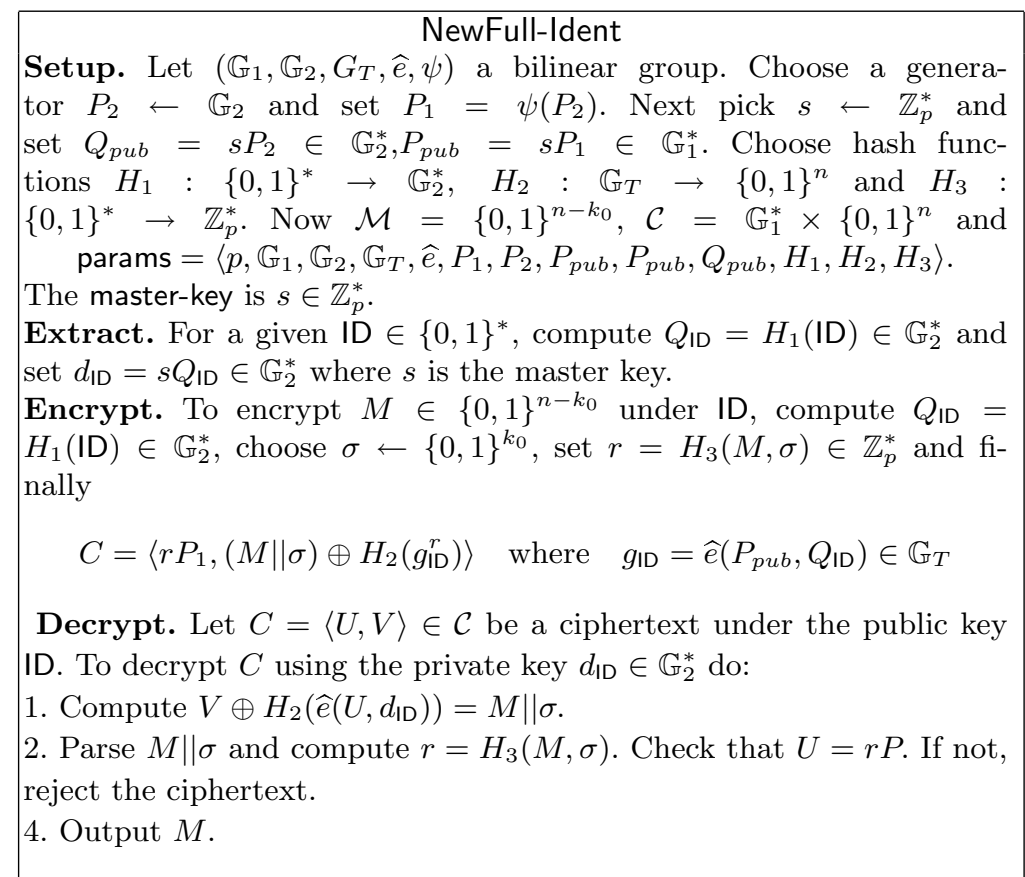

On the basis of the proof sketched in the previous section, we define in a similar fashion a public key encryption scheme NewBasicPub ${ }^{h y}$, which is obtained applying the conversion from expression (3) to Basic-Pub. Then the following results hold:

Result 5 Let $\mathcal{A}_{1}$ an IND-ID-CCA adversary with advantage $\varepsilon_{1}$ against NewFull-Ident making at most $q_{E}$ private key extraction queries, $q_{D}$ decryption queries and $q_{H_{1}}$ hash queries. Then there is an IND-CCA adversary $\mathcal{A}_{2}$ that has advantage at least $\frac{\varepsilon}{q_{H_{1}}}\left(1-\frac{q_{E}}{q_{H_{1}}}\right) \approx \frac{\varepsilon}{q_{H_{1}}}$ against NewBasicPub ${ }^{H y}$. Its running time is $t_{2} \leq t_{1}+c_{\mathbb{G}_{1}}\left(q_{D}+q_{H_{1}}+q_{E}\right)$.

Proof: Use the same reduction as for Result 4 in Section 3.3.

Result 6 Let $\mathcal{A}_{2}$ an IND-CCA adversary with advantage $\varepsilon_{2}$ against NewBasicPub ${ }^{\text {hy }}$ making at most $q_{D}$ decryption queries and at most $q_{H_{2}}$ hash queries. Then there is an IND-CPA adversary $\mathcal{A}_{3}$ that has advantage at least

$$
\left(\varepsilon_{2}-q_{H_{2}} \cdot 2^{-\left(k_{0}-1\right)}\right)\left(1-\frac{1}{p}\right)^{q_{D}} \approx \varepsilon_{2}
$$

against BasicPub. Its running time is $t_{3} \leq t_{2}+q_{H_{2}}\left(T_{\text {BasicPub }}+\log p\right)$, where $T_{\mathrm{Basic}} \mathrm{ub}$ is the running time of Encrypt algorithm in BasicPub. 
Proof: This result is obtained as a special case of Theorem 5.4 in [FO00].

Finally, taking into account these new reductions, we obtain that NewFullIdent scheme is $\left(t_{1}, q_{H}, q_{D}, \varepsilon_{1}\right)$ IND-ID-CCA secure if the BDH problem on $\left(\mathbb{G}_{1}, \mathbb{G}_{2}\right)$ is

$$
\left(t_{1}+c_{\mathbb{G}_{1}}\left(2 q_{D}+q_{H}\right)+q_{H} \mathcal{O}\left(\log ^{3} p+\log p\right), \frac{\varepsilon_{1}}{q_{H}^{2}}\right) \text {-secure }
$$

The last expression has been simplified replacing any of the hash queries $q_{H_{i}}$ by $q_{H}$ and setting $q_{D}=q_{E}$. Then, we get rid of a $q_{H}$ factor in the BDH advantage with respect to the reduction in expression (2).

Compared to Full-Ident scheme, which is the result of using FO transformation in expression (1), the NewFull-Ident scheme presents several advantages:

- It provides more compact ciphertexts. In fact, Full-Ident scheme adds a $n$-bits component to a Basic-Ident ciphertext to get chosen ciphertext security, while NewFull-Ident achieves this preserving Basic-Ident ciphertext's structure.

- It presents a tighter security reduction to the BDH problem.

- It uses one less hash function than Full-Ident.

We can obtain a second tightness improvement using a stronger assumption, namely, the BDDH assumption. In this case, we have the following result:

Result 7 Let $\mathcal{A}_{3}$ an IND-CPA adversary that has advantage $\varepsilon_{3}$ against BasicPub making at most $q_{\mathrm{H}_{2}}$ hash queries. Then there is an algorithm $\mathcal{B}$ breaking the $\mathrm{BDDH}$ problem on $\left(\mathbb{G}_{1}, \mathbb{G}_{2}\right)$ with advantage roughly $\varepsilon_{3}$ and running time $t^{\prime} \approx t_{3}$.

Proof: See Appendix B.

With this second tightness improvement, we obtain that NewFull-Ident scheme is $\left(t_{1}, q_{H}, q_{D}, \varepsilon_{1}\right)$ IND-ID-CCA secure if the BDDH problem on $\left(\mathbb{G}_{1}, \mathbb{G}_{2}\right)$ is

$$
\left(t_{1}+c_{\mathbb{G}_{1}}\left(2 q_{D}+q_{H}\right)+q_{H} \mathcal{O}\left(\log ^{3} q+\log q\right), \frac{\varepsilon_{1}}{q_{H}}\right) \text {-secure }
$$

Then, we get rid of a $q_{H}$ factor in the security reduction at the cost of relying on a stronger assumption.

\section{Conclusions}

In this work, we have shown there is a flawed step in the security reduction exhibited in [BF03] for the so called Boneh-Franklin IBE scheme. We have provided a new reduction without modifying the original scheme neither the underlying hard problem used to state the security.

In the second place, we have proposed a new IBE scheme slightly changing the original scheme. The proposal presents a tighter reduction than BF scheme, uses one less random oracle and has more compact ciphertexts. 
Finally, we point out that it is still an open problem to design an IND-IDCCA IBE scheme with a tight security reduction under a reasonable assumption either in the standard or the random oracle models.

Acknowledgements. The author is grateful to Javier Herranz and Paz Morillo for useful comments on an early draft of this paper. The author also acknowledges the anonymous referees' remarks.

\section{References}

[AP03] S. AlRiyami and K.G. Paterson. Certificateless public key cryptography. In Advances in Cryptology - ASIACRYPT 2003, vol. 2894 of LNCS, pp. 452-473, 2003. Full version available at http://eprint.iacr.org/.

[BDPR98] M. Bellare, A. Desai, D. Pointcheval and P. Rogaway. Relations among notions of security for public-key encryption schemes. In Advances in Cryptology - CRYPTO 1998, vol. 1462 of LNCS, pp. 26-45, 1998.

[BF03] D. Boneh and M. Franklin. Identity-Based encryption from the Weil pairing. SIAM Journal of Computing, 32(3):586-615, 2003. This is the full version of an extended abstract of the same title presented at Crypto'01.

[CC05] Z. Cheng and R. Comley. Efficient certificateless public key encryption. Cryptology ePrint Archive, Report 2005/012, 2005. http://eprint.iacr.org/.

[FO99] E. Fujisaki and T. Okamoto. Secure integration of asymmetric and symmetric encryption schemes. In Advances in Cryptology - CRYPTO 1999, vol. 1666 of $L N C S$, pp. 537-554, 1999.

[FO00] E. Fujisaki and T. Okamoto. How to enhance the security of public-key encryption at minimum cost. IEICE Trans. Fundamentals, E83-9(1):24-32, 2000. This is the full version of [?].

[Gen03] C. Gentry. Certificate-based encryption and the certificate revocation problem. In Advances in Cryptology - EUROCRYPT 2003, vol. 2656 of Lecture Notes in Computer Science, pp. 272-293, 2003.

[GS02] C. Gentry and A. Silverberg. Hierarchical ID-based cryptography. In $A d$ vances in Cryptology - ASIACRYPT 2002, vol. 2501 of LNCS, pp. 548-566, 2002.

[HL02] J. Horwitz and B. Lynn. Toward hierarchical identity-based encryption. In Advances in Cryptology - EUROCRYPT 2002, vol. 2332 of Lecture Notes in Computer Science, pp. 466-481, 2002.

[Sha85] A. Shamir. Identity-based cryptosystems and signature schemes. In $A d$ vances in Cryptology - CRYPTO 1984, vol. 196 of LNCS, pp. 47-53, 1985.

[YFDL04] D. Yao, N. Fazio, Y. Dodis and A. Lysyanskaya. Id-based encryption for complex hierarchies with applications to forward security and broadcast encryption. In Proceedings of the 11th ACM CCS, pp. 354-363. ACM Press, 2004 .

\section{A Proof of Result 4}

If algorithm $\mathcal{B}$ does not abort during the simulation, $\mathcal{A}$ 's view is identical to its view in a real attack: $H_{1}$ behaves as random oracle, and extraction as well as 
decryption queries are valid. Therefore, $\left|\operatorname{Pr}\left[\beta^{\prime}=\beta\right]-1 / 2\right| \geq \varepsilon$, where this probability is over the random bits of $\mathcal{A}, \mathcal{B}$ and the challenger for the IND-ID-CCA game.

It remains to bound the probability $\operatorname{Pr}[\mathcal{B}$ does not abort]. The algorithm can abort for two reasons: (1) it is asked in Phase 1 for the private key query corresponding to $I \mathrm{I}_{j}$, or $(2)$ the challenge identity $I \mathrm{I}_{\mathrm{ch}} \neq \mathrm{ID}_{j}$. Note that $\mathcal{B}$ can not abort in Phase 2 , since in this case $\mathcal{A}$ is not allowed to query the private key for $\mathrm{ID}_{j}=\mathrm{ID}_{\mathrm{ch}}$. Let $\mathcal{E}_{1}$ be the event that $\mathcal{B}$ aborts due to (1), and define $\mathcal{E}_{2}$ in the obvious way.

Then, $\operatorname{Pr}[\mathcal{B}$ does not abort $]=\operatorname{Pr}\left[\neg \mathcal{E}_{1} \wedge \neg \mathcal{E}_{2}\right]=\operatorname{Pr}\left[\neg \mathcal{E}_{2} \mid \neg \mathcal{E}_{1}\right] \operatorname{Pr}\left[\neg \mathcal{E}_{1}\right]$.

We can upper bound for $\operatorname{Pr}\left[\mathcal{E}_{1}\right] \leq q_{E} / q_{H}$, which is the probability that $\mathcal{A}$ makes a extraction query for $\mathrm{ID}_{j}$ in Phase 1 , since the maximum number of such queries is $q_{E}$.

On the other hand, a lower bound for $\operatorname{Pr}\left[\neg \mathcal{E}_{2} \mid \neg \mathcal{E}_{1}\right]$, that is the probability that $\mathcal{A}$ chooses $\mathrm{ID}_{j}$ as the challenge identity, is $1 / q_{H_{1}}$. Therefore,

$$
\operatorname{Pr}[\mathcal{B} \text { does not abort }] \geq \frac{1}{q_{H_{1}}}\left(1-\frac{q_{E}}{q_{H_{1}}}\right) .
$$

This shows that $\mathcal{B}^{\prime} s$ advantage is at least

$$
\frac{\varepsilon}{q_{H_{1}}}\left(1-\frac{q_{E}}{q_{H_{1}}}\right) .
$$

\section{B $\quad$ Proof of Result 7}

Algorithm $\mathcal{B}$ receives as inputs the bilinear group $\left(\mathbb{G}_{1}, \mathbb{G}_{2}\right)$, and a random instance $\left(P_{2}, a P_{2}, b P_{2}, c P_{1}, T\right)$ from either $\mathcal{P}_{B D H}$ or $\mathcal{R}_{B D H}$ distributions. Then $\mathcal{B}$ uses $\mathcal{A}$ IND-CPA advantage against BasicPub to distinguish $\mathcal{P}_{B D H}$ from $\mathcal{R}_{B D H}$.

Setup $\mathcal{B}$ provides $\mathcal{A}$ with the public key pk $=\left\langle p, \mathbb{G}_{1}, \mathbb{G}_{2}, \mathbb{G}_{T}, \widehat{e}, P_{1}, P_{2}, P_{\text {pub }}, Q_{\text {pub }}, Q, H_{2}\right\rangle$, where $P_{1}=\psi\left(P_{2}\right), Q_{p u b}=a P_{2}, P_{p u b}=\psi\left(Q_{p u b}\right), Q=b P_{2}$ and $H_{2}$ is a random oracle controlled by $\mathcal{B}$ as explained below. Notice that the unknown private key of BasicPub is $d=a b P_{2}$.

$H_{2}$-queries To respond $\mathcal{A}$ queries to $H_{2}, \mathcal{B}$ maintains a list $H_{2}^{\text {list }}$ of tuples $\left\langle X_{i}, H_{i}\right\rangle$. When queried with $X_{i}$, algorithm $\mathcal{B}$ does the following:

1. If $\left\langle X_{i}, H_{i}\right\rangle \in H_{2}^{\text {list }}$, it returns $H_{2}\left(X_{i}\right)=H_{i}$.

2. Otherwise, $\mathcal{B}$ picks $H_{i} \leftarrow\{0,1\}^{n}$, adds the tuple $\left\langle X_{i}, H_{i}\right\rangle$ to the list and returns $H_{2}\left(X_{i}\right)=H_{i}$.

Challenge $\mathcal{A}$ outputs two equal length plaintexts $M_{0}, M_{1}$ in which it wishes to be challenged. Algorithm $\mathcal{B}$ returns as the challenge ciphertext $C=\left\langle c P_{1}, M_{\beta} \oplus\right.$ $\left.H_{2}(T)\right\rangle$, where $\beta \leftarrow\{0,1\}$.

Guess $\mathcal{A}$ eventually outputs a guess $\beta^{\prime}$ for $\beta$. Algorithm $\mathcal{B}$ returns 1 if $\beta^{\prime}=\beta$ and 0 otherwise. 
Algorithm $\mathcal{B}$ is simulating a real attack environment for $\mathcal{A}$. If the random instance is from $\mathcal{R}_{B D H}$, then $\operatorname{Pr}\left[\beta^{\prime}=\beta\right]=1 / 2$, since in this case the distribution of the ciphertext $C$ is independent of the bit $\beta$. Otherwise, the instance comes from $\mathcal{P}_{B D H}, C$ is a valid encryption of $M_{\beta}$ and therefore $\operatorname{Pr}\left[\beta^{\prime}=\beta\right]=1 / 2+\varepsilon$ by definition of $\mathcal{A}$. Therefore,

$$
\left|\operatorname{Pr}\left[\mathcal{B}\left(\mathcal{P}_{B D H}\right)=1\right]-\operatorname{Pr}\left[\mathcal{B}\left(\mathcal{R}_{B D H}\right)=1\right]\right|=|1 / 2+\varepsilon-1 / 2|=\varepsilon .
$$

